\title{
CAVAL REFLUXES IN ANGIOCARDIOGRAPHY AND THE DYNAMICS OF THE RIGHT ATRIUM
}

\author{
BY \\ CHRISTIAN HEDMAN, JOHN LIND, AND CARL WEGELIUS \\ From the Pediatric Clinic of the Caroline Institute (Prof. A. Wallgren) and the Department for Cardio- \\ vascular Research of the Wenner-Gren Institute, Stockholm
}

Received November 22, 1952

During the past few years angiocardiography has become one of the routine methods in the investigation of congenital heart disease. The development of the technique has increased its diagnostic value so that it offers a method of judging not only the anatomical, but also the pathophysiological aspects of a heart disease. This paper deals with a problem often met with in angiocardiography, i.e. the regurgitation of dye from the right atrium into the venæ cavæ and their proximal branches, and its relation to the function of the right atrium. This phenomenon, which occurs especially in children, has sometimes been thought to have a special diagnostic value. Up to the present, however, no detailed investigation of the mechanism of this reflux appears to have been carried out.

\section{Angiocardiographic Technique Used in the Present Investigation}

The development of fast direct angiocardiography (10-12 exp./sec.) in two planes at an angle of $90^{\circ}$, with synchronous electrocardiographic recording, enables us not only to study the morphology but also to gain physiological and patho-physiological information, regarding the dynamics of the heart and may thus offer us a way of approaching the problem. The following is an account of some observations, using this method in cases with or without cardiac lesions interfering with the function of the right heart.

We have used the following amounts of contrast medium (umbradil, astra) in a 70 per cent solution, given intravenously.

\begin{tabular}{ccc} 
Weight in $\mathrm{kg}$. & \multicolumn{2}{c}{ Dose, ml./kg. of weight } \\
& $\begin{array}{c}\text { Injection in } \\
\text { cubital vein }\end{array}$ & $\begin{array}{c}\text { Injection in } \\
\text { malleolar vein }\end{array}$ \\
$2.5-5$ & $1.00-1.5$ & $1.25-1.50$ \\
$5-10$ & $1.00-1.25$ & 1.25 \\
$10-20$ & $0.75-1.25$ & $1.00-1.25$
\end{tabular}

Within these limits, the amount given is governed by the cardiac volume. Satisfactory roentgenographic contrast, providing good or adequate visualization of all the heart cavities and the large vessels, can usually be obtained with these amounts of contrast medium. The speed of the injection has, as far as possible, been kept constant.

All children received a 2.5 per cent solution of avertin, $0 \cdot 1 \mathrm{ml}$. per kilogram of weight by rectum and remained quiet, with shallow respiration, during the examination.

\section{Anatomy and Physiology of the Vene Cave}

It seems reasonable that the orifices of the great systemic veins that terminate in the right atrium must close at the start of each atrial systole. Otherwise some of the force of the contraction would be spent in returning blood to the veins. Careful investigations have been carried out concerning the mechanism of closure of caval veins.

The opening of the superior vena cava, devoid of a valve, is situated at the upper and posterior part of the cavity. The orifice is elliptical in shape with a right anterior and left posterior margin (which are brought in contact with one another at the start of each atrial systole, thus closing the orifice). Among the muscle fibres peculiar to each atrium are annular fibres surrounding the extremities of the large vessels, as they 
open into the atrium. Keith (1902) has especially studied the structures that close the venous orifices of the right atrium and according to him four muscular bands bring this about (tænia terminalis, superior and inferior limbic bands, and the septal band). Further, the terminal half inch of the superior vena cava is surrounded by loops of muscle fibres which are regarded as the only representative of the musculature of the sinus venosus now remaining in the mammalian heart.

The orifice of the inferior vena cava is situated at the lower and posterior part of the atrium and bounded anteriorly by the vestigal valve of the inferior vena cava. In addition, there are annular muscle fibres present which surround the opening.

According to experimental evidence the venous orifices remain patent during atrial systole and the physical forces that prevent regurgitation are the following (Wiggers, 1949): (a) the necessity of stopping the onflowing blood before the stream can be reversed, $(b)$ the fact that pressure is lower in the ventricles than in the veins so that the atria tend to empty in the direction of least resistance, $(c)$ the peristaltic mode of contraction which favours emptying towards the ventricles, and $(d)$ the emptying of the atria during early ventricular diastole so that they contain but a small quantity of blood when the atria contract.

\section{ANGIOCARDIOGRAPHIC OBSERVATIONS}

Dynamics of the right atrium. The right atrium contracts mainly in the antero-posterior direction, the reduction in height being less marked. This agrees with the anatomical finding that the lateral fornices of the caval veins are connected by the sulcus terminalis, an " indifferently marked fibrous septum" (Keith, 1902). The distance between the lateral fornices of the caval orifices is, therefore, scarcely altered in contraction of the atrium. A peristaltic mode of contraction has not been observed. The contraction starts at the summit of the $P$ wave and the atrium is small at the $\mathrm{Q}$ wave in the synchronous electrocardiogram. It seems to become slightly smaller during the $\mathrm{Q}-\mathrm{S}$ interval-perhaps due to the closure of the tricuspid valve. The refilling of the atrium begins about a hundredth of a second after the $S$ wave. It is rapid to start with, but slow from the end of the T wave to the $P$ wave. Sometimes we have noticed some reduction in its size just after the end of the $\mathrm{T}$ wave due to the passage of blood into the right ventricle, the A-V valve opening at this point. In infants with a rapid heart rate (120-180 a minute) the A-V valve seems, however, to remain closed till the atrium contracts, indicating that the pressure in the right atrium only then becomes greater than in the right ventricle. The filling of the ventricle in these cases thus occurs during the short interval from the point of the $P$ wave to the $Q$ wave.

The dynamic importance of atrial contraction has been much debated. It has been demonstrated that filling of the ventricle occurs mainly during early diastole, due to the difference in atrial and ventricular pressure at the end of the isometric relaxation. In infants, with their rapid heart rates the atrial contraction seems, however, to be the chief force that fills the ventricle.

The relation of the size of the right atrium in maximum atrial systole and diastole, as seen in angiocardiograms, is approximately $1: 3$ or $1: 4$ (Fig. 1).

\section{Refluxes of Contrast during Injection via the Superior Vena Cava}

If the dye reaches the orifice of the superior vena cava during atrial systole it is stopped. The calibre of the vein, except in the proximal part, increases and sometimes there is retrograde filling of its small tributaries. The most proximal part of the vein, however, shows a circular narrowing during atrial systole. It appears in most cases even during the injection of contrast medium and seems, therefore, to be an indication that this portion of the vein takes an active part in the atrial contraction (Fig. 1).

When the atrium has become contrast-filled through the superior vena cava- sometimes see, even in normal cases, dye leaving the atrium through the inferior vena cava. This hapens only during atrial diastole when the orifices of the caval veins are not closed.

In the pathological conditions that interfere with the function of the right side of the heart, for instance atrial or ventricular septal defect, refluxes are regularly seen down into the inferior vena cava. They are seen also during atrial systole, from the peak of the $P$ wave to just after the $S$ wave but they occur most often and have their maximum intensity in the latter half of the interval (Fig. 2). 


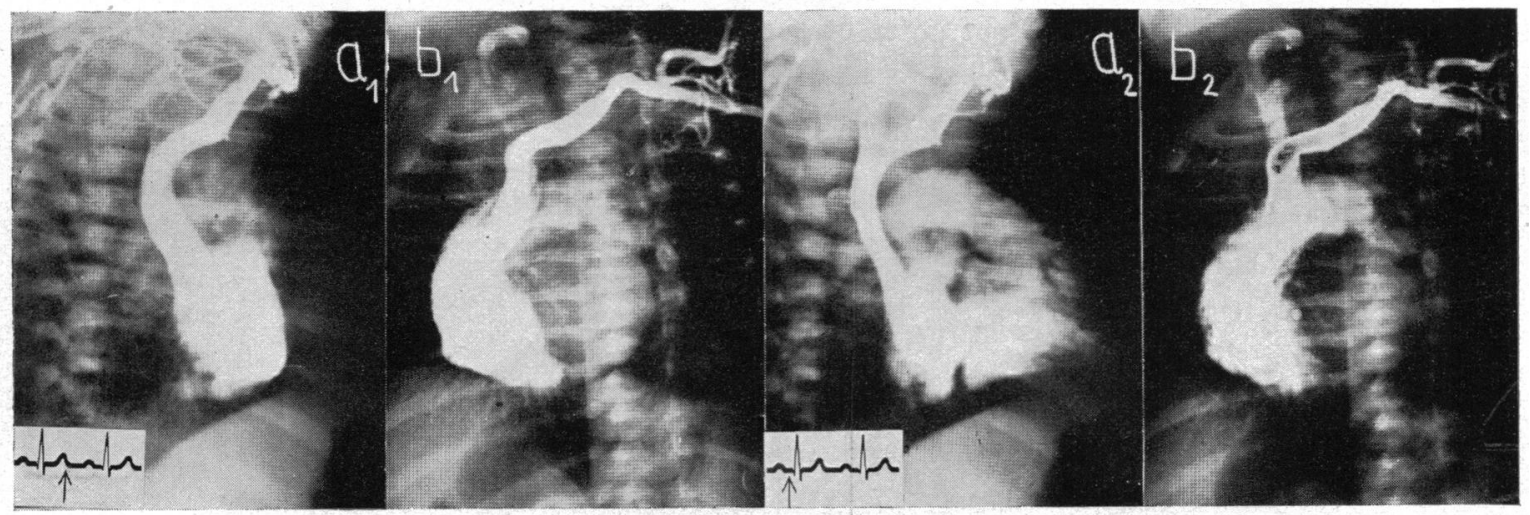

Fig. 1.-Narrowing of the superior vena cava simultaneously with atrial systole. Figures $a_{1}, a_{2}$ in the right anterior oblique and $b_{1}, b_{2}$ in the left anterior oblique position. $a_{1} b_{1}$ : The atrium is seen to be biconvex. The superior vena cava is broad. $\mathbf{a}_{2} \mathbf{b}_{2}$ : During atrial systole the superior vena cava contracts, as demonstrated by a marked diminution in the calibre of the proximal part of the vessel.

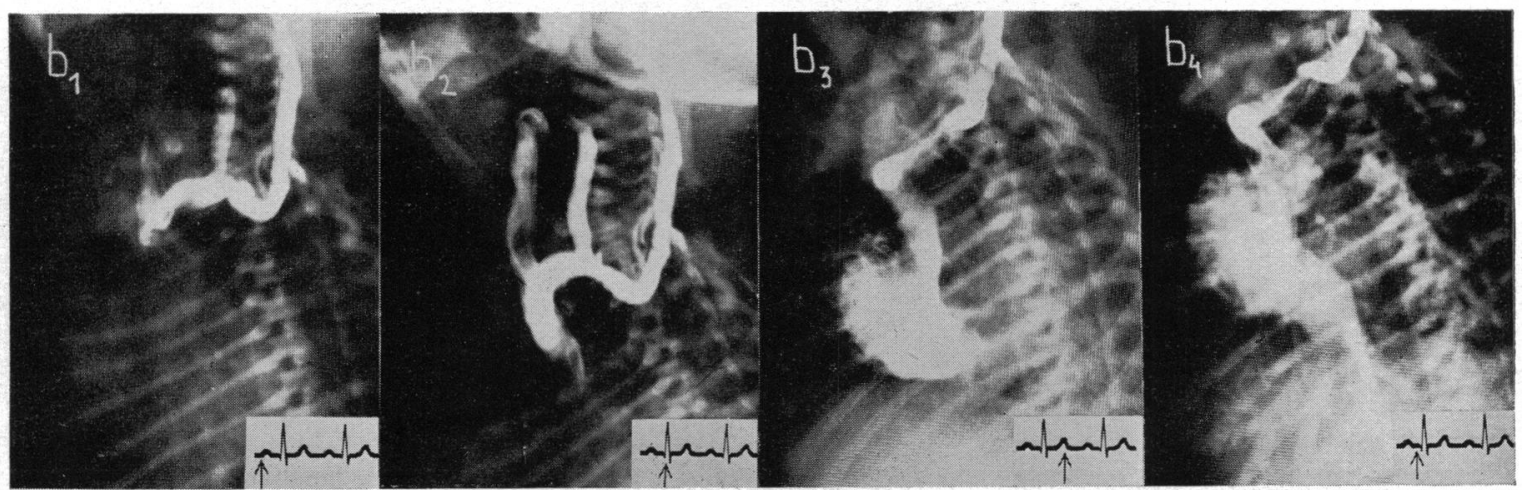

FIG. 2.-The closure of the orifice of the superior vena cava and demonstration of systolic reflux downwards into the inferior vena cava in a case of atrial septal defect with left to right shunt.

Injection of contrast medium is done through an antecubital vein.

Left anterior oblique position.

$b_{1}$ : The dye on its way through the left innominate vein. Beginning of atrial systole with a retrograde filling of the small venous tributaries.

$b_{2}$ : End of the same atrial systole. The dye has reached the closed orifice of the superior vena cava. Large refluxes of dye up into the internal jugular veins.

$b_{3}$ : One-tenth of a second later. The right atrium becomes opacified instantly with the contrast medium which has accumulated in the afferent veins.

$\mathbf{b}_{4}$ : In the following atrial systole there is a large reflux of diluted dye downwards into the hepatic veins. The dilution of dye in the right atrium may be due to a left to right atrial shunt.

\section{Refluxes of Contrast during Injection via the Inferior Vena Cava}

Here the contrast medium reaches the right atrium from below and if it arrives at the orifice during atrial systole it is held up and directed downwards into the hepatic veins (Fig. 3).

In contrast with the superior vena cava the proximal part becomes dilated in atrial systole and thin in diastole when the passage into the atrium is free.

When the atrium is heavily contrast filled, i.e. dye has filled the atrium during the whole of diastole, dye sometimes passes upwards into the superior vena cava during the end of diastole, 
even in normal cases (Fig. 4). In atrial or ventricular septal defects, with left-to-right shunts, systolic refluxes downwards into the inferior vena cava are a regular finding. Refluxes up into the superior vena cava are less common. In relation to the electrocardiogram they occur during the interval from the top of $P$ to just after the $S$ wave, the maximum regurgitation taking place at the end of the P-S interval.

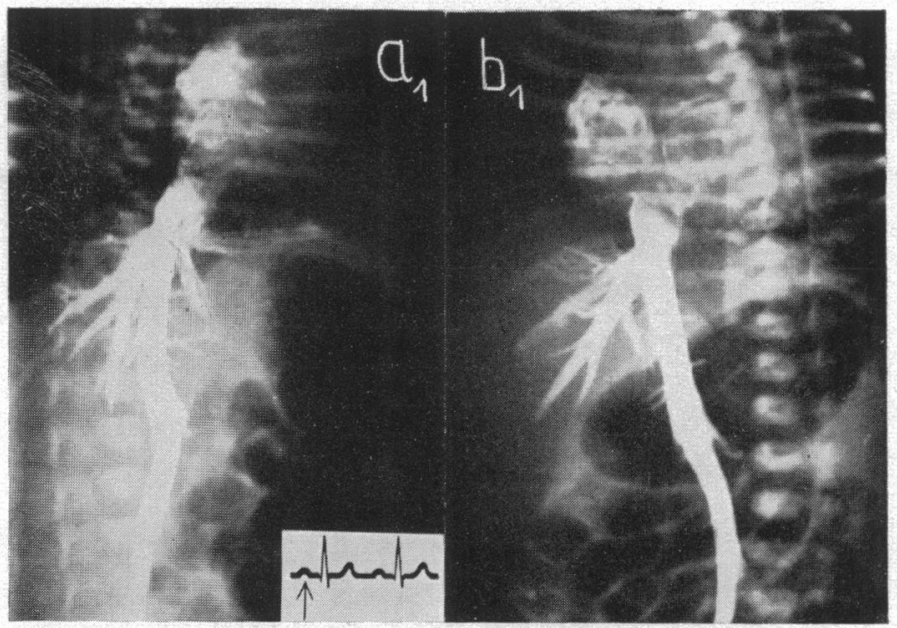

Fig. 3.-Closure of the inferior vena cava and demonstration of a systolic "pseudo-reflux" into the hepatic veins. Injection of dye into a malleolar vein.

The dye has reached the orifice of the inferior vena cava during atrial systole. No dye enters the atrium. The vena cava is wide and some dye is diverted downwards into the hepatic veins.

$b_{1}$ : In $b_{1}$ it can be seen that the dye is about equally distributed between the right and left atria, due to an atrial septal defect.

$a_{1}$ : In $a_{1}$ it is seen that only an insignificant amount of dye has so far passed into the ventricles.

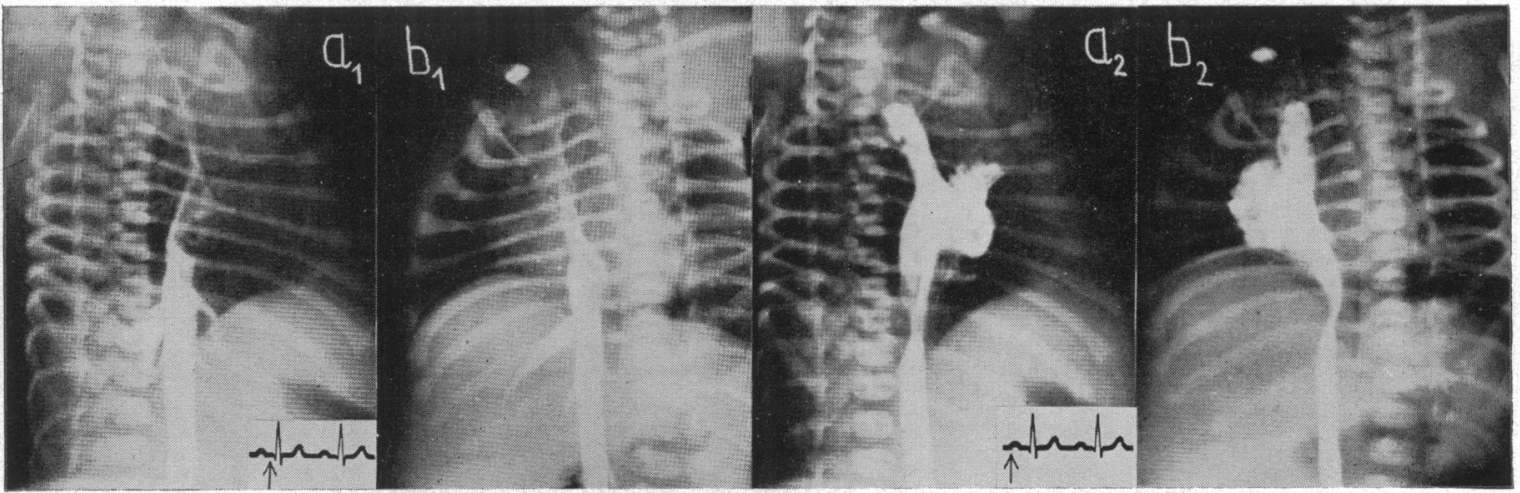

Fig. 4.-Closure of the inferior vena cava and demonstration of a reflux during atrial diastole.

Injection of dye into a malleolar vein.

$a_{1}, a_{2}$ in right anterior oblique, $b_{1}, b_{2}$ in left anterior oblique position.

$a_{1} b_{1}$ : The inferior vena cava is broad in atrial systole. Its orifice is closed, permitting only a fine stream of dye to enter the atrium.

$a_{2} b_{2}$ : Right atrium is densely opacified. The large auricular appendage is seen. Dye passes out in a broad stream through the open orifice of the superior vena cava.

Note that the superior vena cava is broad and the inferior vena cava thin in atrial diastole. 


\section{Discussion}

The refluxes seen during angiocardiography are of essentially different origins.

Pseudo-refiuxes. Here the contrast medium never has been in the atrium. Due to the closure of the caval orifice during atrial systole the dye is held up and there occurs a retrograde filling of the venous tributaries. These refluxes are seen in normal hearts as well as in all sorts of congenital cardiac lesions.

True Refluxes. Here the dye leaves the right atrium through one of the venæ cavæ. They occur most often downwards into the inferior vena cava, which is in agreement with clinical experience and anatomical investigations that have shown that this orifice is the weakest point of the right atrium.

Refluxes occurring in Atrial Diastole. These are often seen in congenital malformations of the heart with increased right atrial pressure and sometimes even in normal hearts. The mechanism seems to be an overloading of the atrium with contrast medium from the injection. Consequently, at the end of atrial diastole before contraction has started, the atrial pressure becomes higher than in the vena cava not used for injection and some dye passes out through its open orifice.

Cardiac catheterization studies in adults have indicated that rapid injections of dye do not significantly alter the right atrial pressure. In view of the short distance between the site of injection and the right atrium in small children and infants, an elevation of the atrial pressure may, however, be possible. In children the amount of contrast medium used is relatively greater than in adults. In atrial diastole the open caval orifices seem, however, to serve as safety valves and thus the increase in atrial pressure during angiocardiography may be small, if perceptible.

There appears to be little doubt that these refluxes, as well as the pseudo-refluxes, are produced by the injection of dye. They are consequently more frequent the younger the infant and the greater the amount of dye injected per second. They disappear rapidly and occur only during and immediately after the injection. It is obvious that the synchronously recorded electrocardiogram is of great importance for the identification of these refluxes as well as the pseudo-refluxes.

Refluxes occurring in Atrial Systole. In most cases true refluxes, however, occur during and most commonly at the end of atrial contraction. This may be explained by the fact that the start of effective mechanical contraction corresponds to the summit of the $P$ wave in the simultaneous electrocardiogram, but the maximum pressure is not reached until about $0.02 \mathrm{sec}$. later. Further, it is seen that the right ventricle becomes rapidly filled in early diastole to a point beyond which further distension may be resisted. That refluxes are noted corresponding to the Q-S intervals may be due to the fact that shortly after the onset of ventricular contraction the atrial pressure rises abruptly, following closure of the A-V valve, which bulges into the atrium. It is probable that the course of the atrial pressure curve depends upon the heart rate and the contribution by atrial systole to ventricular filling.

By using optical manometers to record pressure changes in the atria and ventricles it has been found that the maximum atrial pressure coincides with the isometric contraction of the ventricles (Wiggers, 1948). By catheterization of the heart the atrial pressure is found to be lower at the beginning of the isometric contraction of the ventricle than at the start of the atrial systole, and that the isometric ventricular contraction produces only a little quick and sharp rise in the pressure (Lagerlöf and Werkö, 1948). The presence of the catheter, introduced through one of the caval orifices may, however, modify the normal pressure curve. None of the methods seem, therefore, able to give exact information about the atrial pressure variations.

Refluxes of dye during atrial systole are not seen in normal cases. In this phase of the heart cycle the caval orifices are closed and no dye is entering or leaving the heart. The increase in diastolic filling due to the injection is not followed by an increase in the systolic atrial pressure sufficient to break the closure mechanism of the caval orifices.

In congenital heart lesions where there is an extra load on the right atrium, as in atrial or ventricular septal defects with significant left-to-right shunts, tetralogy of Fallot, etc., systolic 
refluxes are on the other hand regularly seen-even when a small amount of dye is used (Fig. 5 and 6). In angiocardiograms from such cases the refluxes are seen to occur not only in the contrastloaded heart cycles during the injection but for quite a long time afterwards. They have a diagnostic value, representing a visualization of a patho-physiologic phenomenon.

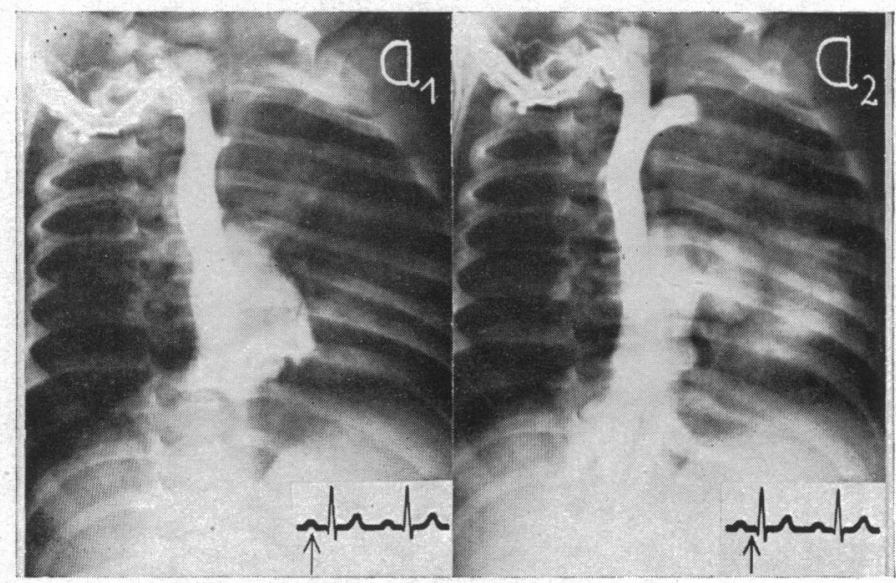

FIG. 5.-Incompetence of the inferior caval orifice in a case of tricuspid atresia.

Injection of dye through the right antecubital vein.

Right anterior oblique position.

$\mathrm{a}_{1}$ : Atrial diastole. The superior vena cava and right atrium are filled with contrast medium.

$a_{2}$ : During atrial systole there is a heavy reflux downwards into the dilated inferior vena cava and hepatic veins.

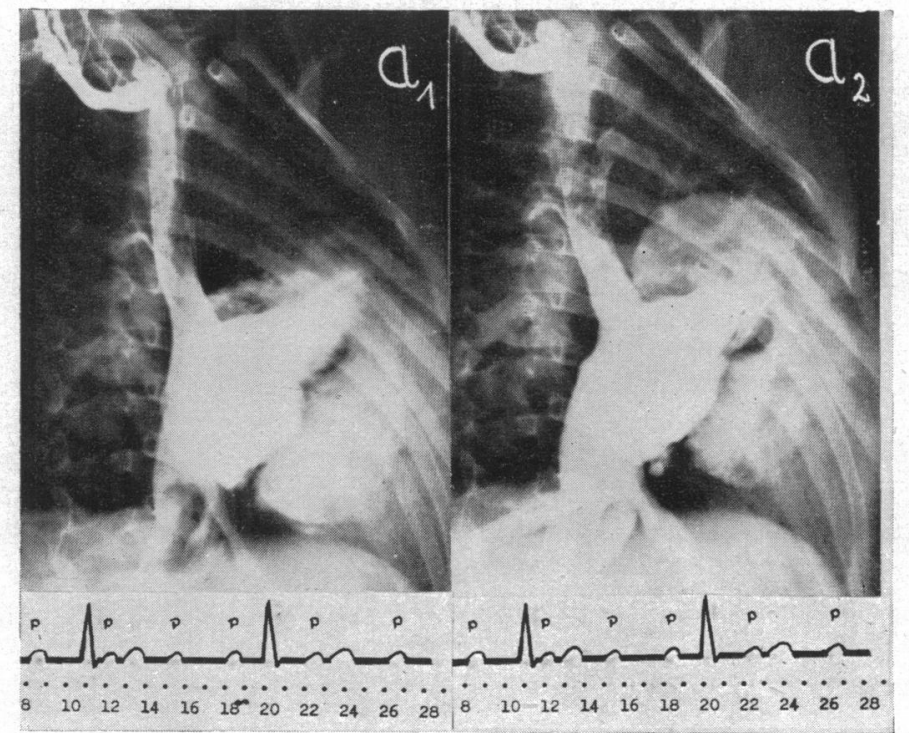

Fig. 6.-Simultaneous atrial and ventricular systole in a case of complete heart block.

Injection of contrast medium is done through the right antecubital vein.

Right anterior oblique position.

$\mathrm{a}_{1}$. Right atrium in diastole, right ventricle in systole (exposure $\mathrm{nr} .11$ in the accompanying electrocardiogram).

$a_{2}$ : Atrial systole occurs before the ventricular contraction is finished. Heavy reflux occurs downwards into the inferior vena cava, but there is also a retrograde filling of the superior vena cava. Sinus venosus is also seen opacified in the atrio-ventricular groove (exposure nr. 13 in the accompanying electrocardiogram). 
The explanation for their existence in these cases is complex. There is often a dilation of the right atrium perhaps combined with a weakening of the closure-mechanism of the caval orifices.

Further, there is an increased atrial pressure, which may become even higher following the injection and reach the critical level of resistance of the orifices. Nearly always, however, the entrance of the dye is stopped during atrial systole.

Other factors possibly involved in the origin of refluxes must be considered. No specific effect of the contrast medium on the myocardium can be expected during the observation period which lasted only up to 1 second after dye has reached the heart. Such effects are met with 1-2 seconds later. Neither does respiration seem to have any influence on the reflux mechanism in our study. All children were well anæsthetized. They were completely quiet during angiocardiography (with shallow regular respiration). The interval between the films, i.e. the observations, are also very short. Therefore changes in the intrathoracic pressure must be small and may be considered negligible.

\section{SUMMARY}

Refluxes of contrast medium from the right atrium into the venæ cavæ and their relation to the dynamics of the right atrium have been studied by fast angiocardiography (10-12 pictures a second in two projections at right angles) with simultaneous electrocardiographic registration. It has been shown that the caval orifices are normally closed during atrial systole. The refluxes have been classified according to their origin. Their significance is discussed with emphasis on the importance of simultaneous electrocardiographic registration for diagnostic differentiation.

\section{REFERENCES}

Cunningham, D. J., ed. by Brash, J. C. (1951). Textbook of Anatomy. 9th ed., Oxford University Press, London. Dotter, C. T., and Steinberg, I. (1951). Angiocardiography. Annals of Roentgenology, Vol. XX. Paul B. Hoeber Inc., New York.

Keith, A. (1902). Proc. Anat. Soc. Great Brit. and Ireland, p. 2.

Lagerlöf, H., and Werkö, L. (1948). Cardiologia, 13, 241.

Lind, J., and Wegelius, C. (1952). Adv. in Ped., 5, 154.

Wiggers, C. J. (1949). Physiology in Health and Disease. 5th ed., Lea \& Febiger, Philadelphia. 